Revue d'histoire de l'Amérique française

REVUE D.HISTOIRE DE L'AMÉRIQUE FRANÇAISE

\title{
Un nouvel inventaire du Saint-Laurent, 1603
}

\section{Marcel Trudel}

Volume 16, numéro 3, décembre 1962

URI : https://id.erudit.org/iderudit/302209ar

DOI : https://doi.org/10.7202/302209ar

Aller au sommaire du numéro

Éditeur(s)

Institut d'histoire de l'Amérique française

ISSN

0035-2357 (imprimé)

1492-1383 (numérique)

Découvrir la revue

Citer cet article

Trudel, M. (1962). Un nouvel inventaire du Saint-Laurent, 1603. Revue d'histoire de l'Amérique française, 16(3), 313-347. https://doi.org/10.7202/302209ar d'utilisation que vous pouvez consulter en ligne.

https://apropos.erudit.org/fr/usagers/politique-dutilisation/ 


\title{
UN NOUVEL INVENTAIRE DU SAINT-LAURENT, 1603 *
}

\begin{abstract}
Une révolution ethnique dans le Saint-Laurent. La toponymie se donne un nouveau visage. La brève carrière américaine d'Aymar de Chaste. Un observateur, Samuel de Champlain. La «tabagie» de Tadoussac. Champlain remonte le Saint-Laurent. De nouveau, le mystère de l'horizon occidental. Une nouvelle séduction, l'Acadie. Le bilan du voyage de 1603. L'Angleterre n'est pas plus heureuse.
\end{abstract}

Les préoccupations du commerce ont ramené la France au nord de l'Amérique et, comme si elle n'avait rien pu retenir des tentatives de Cartier et de Roberval, elle s'arrête à des expériences qui ne l'approchent guère de son objectif, une NouvelleFrance: le marquis de La Roche s'installe sur un îlot sablonneux à 90 milles de la terre ferme; Chauvin remonte le Saint-Laurent, mais c'est pour s'établir en un point stérile, Tadoussac, où ne peut tenir qu'un comptoir saisonnier. Pourtant, nous sommes en 1602, on travaille depuis plus de soixante ans à provigner une France nouvelle: elle n'est pas établie; on ne sait même pas encore où l'établir. Il semble, cependant, que la grande voie de pénétration, découverte par Cartier en 1535, ait des chances de devenir enfin le choix définitif.

* Extrait d'un volume qui paraîtra bientôt, Histoire de la NouvelleFrance, 1524-1603. 


\section{UNE REVOLUTION ETHNIQUE DANS LE SAINT-LAURENT}

La grande vallée du Saint-Laurent a bien changé depuis 1543.

Du temps de Cartier et de Roberval, elle était le domaine des Iroquois, établis sur l'île stratégique d'Hochelaga, à Achelacy, à Stadaconé et dans plusieurs petits villages des environs. Entretenant entre eux une unité assez lâche, les Iroquois contrôlaient tout le fleuve jusqu'au golfe où ils allaient, du moins ceux de Stadaconé, faire leurs pêches. Certes, ils avaient des ennemis: les Toudamans, qui seraient les Micmacs et les Etchemins, les Agojudas, en qui on croit reconnaître les Algonquins $\mathrm{du}$ haut de la rivière des Outaouais. La position de ces Iroquois laurentiens semblait si assurée qu'ils n'ont pas craint de faire à Cartier une lutte politique d'abord, qui amène l'exil de Donnacona au bénéfice d'Agona, puis une véritable lutte militaire qui coûte des hommes à Cartier et l'oblige à s'éloigner. Les guerres franco-iroquoises étaient commencées.

Or, quand les Français reviennent un demi-siècle plus tard, tout est changé dans le Saint-Laurent. Les rives du fleuve sont désertes, ou peut s'en faut: vers l'île aux Lièvres, Champlain rencontre en 1603 quelques indigènes; en bas de l'île aux Coudres, il note une petite rivière de la rive nord où les sauvages «cabanent » quelquefois; il remarque des Algonquins cabanés vis-àvis l'île d'Orléans; d'autres sauvages ont des cabanes à l'entrée de la rivière Richelieu ${ }^{1}$. On n'a plus affaire qu'à des nomades, il n'y a plus de sédentaires. Champlain arrive à Québec et il n'y voit aucun habitant; il ne fait même pas allusion à l'ancien Stadaconé ni à Achelacy; quand il s'arrête au sault Saint-Louis, il n'écrit pas une seule fois le nom d'Hochelaga ni celui de Tutonaguy. Les cultures et les villages que Cartier avait décrits ont disparu et n'ont pas été remplacés. Les anciens maîtres du Saint-Laurent sont partis: quand Champlain parle des Iroquois, c'est pour les situer bien loin au sud-ouest, vers le lac Ontario et le lac Champlain. Ce désert des rives laurentiennes, au début

${ }^{1}$ Champlain, Oeuvres (éd. Biggar), I: 127, 141, 158s., 162. 
du dix-septième siècle, a même fait mettre en doute la véracité des relations de Cartier $^{2}$ : on ne pouvait imaginer une transformation aussi rapide.

Comment cette révolution s'est-elle produite? Le silence des voyageurs est là-dessus déconcertant. De 1543 à 1603, rien qui puisse nous éclairer sur ce bouleversement ethnographique et politique du Saint-Laurent. Jacques Noël, qui est venu dans le Saint-Laurent vers 1585 , qui a vu les ruines des forts de son oncle Cartier et qui est allé jusqu'à Hochelaga, ne nous a laissé aucun indice; il ne nous dit même pas si les indigènes qu'il a vus sont ceux que Cartier avait connus quarante ans plus tôt. La révolution était-elle déjà commencée ou même terminée ? Il faudrait la situer assez tôt après les voyages de Cartier et de Roberval: Champlain aurait dit que les «anciens du païs» n'avaient jamais rien vu à Hochelaga; selon Lescarbot, qui publie en 1618, les Français qui «hantent aujourd'huy» le SaintLaurent, «n'entendent rien » au dictionnaire que Cartier nous a laissé «du langage de Canada » ${ }^{3}$. Entre Cartier et Lescarbot, il n'y a pourtant qu'un demi-siècle ! Par ailleurs, on pourrait croire que cette révolution est plus récente et daterait des environs de 1600: en 1608, Lescarbot emploie l'expression il $y$ a quelques années ${ }^{4}$.

Ce changement est-il dû à la guerre ? C'est ce qu'affirme Champlain; Lescarbot écrit que les Iroquois furent surpris «dans leur enclos » par huit mille ennemis qui les détruisirent ${ }^{5}$; selon l'auteur de la Relation de 1644, les Algonquins étaient devenus maîtres de leurs ennemis iroquois et les avaient défaits partout ${ }^{6}$; dans un mémoire de 1697 , La Chesnaye précise que, d'après une tradition, les Algonquins « avoient chassé les Iro-

${ }^{2}$ Lescarbot, pour sa part, ajoute foi à cette transformation, bien que \& ledit Champlain et autres disent que jamais il n'y a rien eu [à Hochelaga] et n'y en apparoit aucun vestige, et que anciens du païs rapportent n'avoir jamais rien veu $\gg$; et Lescarbot ajoute que Champlain a vu en 1609 , chez les Iroquois, une « forme de ville du tout semblable » à celle d'Hochelaga (Lescarbot, Histoire de la Nouvelle-France, éd. Tross, III: 606s.)

3 Lescarbot, op. cit., III : 662.

4 Ibid., III : 665.

5 Loc. cit.

${ }^{6}$ Relations des Jésuites (éd. Thwaites), XXV : 107. 
quois du lieu de Quebec et les environs ou estoit autrefois leur demeure; l'on nous montroit leurs bourgades et villages couverts de bois nouveaux jets $\gg 7$. Cette explication est vraisemblable. Déjà, à l'époque de Cartier, les Iroquois devaient lutter contre les Toudamans, et ceux-ci en 1534 leur avaient tué environ 200 personnes dans une île en face du Saguenay; ils avaient aussi une guerre continuelle contre les Agojudas, «armés jusques sus les doidz », portant des armures «qui sont de cordes et de boys, lasséez et tissuez ensemble ${ }^{8}$.

$\mathrm{Au}$ lieu d'une extermination par les ennemis, y eut-il simplement migration des Iroquois ? C'était la coutume chez les nations iroquoises, comme chez d'autres, de déménager à intervalles plus ou moins longs lorsque le sol était épuisé; en 1541, on ne parle déjà plus d'Hochelaga où, six ans plus tôt, Cartier avait été reçu de façon solennelle, mais seulement de Tutonaguy, situé en amont. Pressés par des impératifs économiques et, peut-être en même temps, par un plus grand besoin de sécurité, les Iroquois laurentiens ont pu préférer se retirer à l'intérieur des terres pour rejoindre un peuple de même langue et de même culture.

Selon une hypothèse récente, la disparition des Iroquois laurentiens serait due à cette ligue, consolidée bien avant 1603 , que formaient les Algonquins de la rivière des Outaouais, les Montagnais du Saguenay et les Etchemins de la rive sud. Depuis l'époque de Cartier, le Saint-Laurent était devenu pour les Français un magnifique marché à fourrures; les fournisseurs en sont les peuples chasseurs (Algonquins, Etchemins et Montagnais) à qui une nation sédentaire, celle des Iroquois laurentiens, bloque la route d'eau: Hochelaga oppose un barrage que complètent Achelacy et Stadaconé. Pour échapper au contrôle des Iroquois, les peuples chasseurs recourent à des voies d'évitement, longues et compliquées de portages: route des Algonquins par la Gatineau et route par le Saint-Maurice jusqu'au bassin du lac

7 Cité par A. G. Bailey, The Significance of the Identity and Disappearance of the Laurentian Iroquois, dans MSRC, 27 (1933): 106, n. 50.

8 Relation du deuxième voyage de Cartier, dans Les Français en Amérique pendant la première moitié du XVIe siècle, 152, 156. 


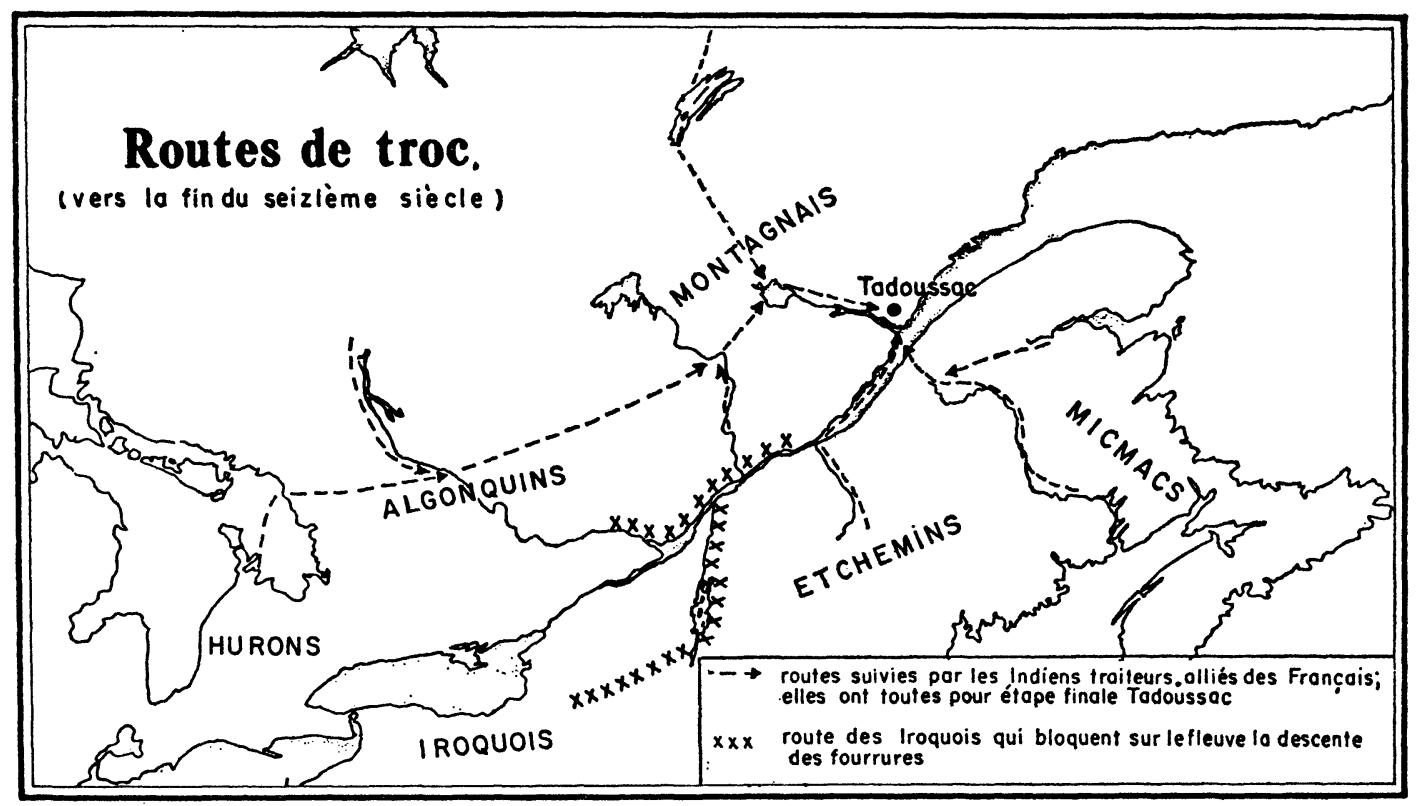

Ces routes aboutissent à Tadoussac où les Français font leurs échanges.

Algonquins et Montagnais voyagent à l'intérieur du pays pour éviter les Iroquois qui viennent faire des incursions dans le Saint-Laurent. Les marchandises françaises atteignent déjà le pays des Hurons. 
Saint-Jean, d'où l'on descend à Tadoussac. De plus, par le troc, les fournitures d'Europe pénètrent rapidement chez les alliés et même, par les Algonquins, jusque chez les «bons Irocois» (qui seraient les Hurons); parmi ces fournitures, il y a le fer dont les nations de la ligue disposent dès le seizième siècle et qui va leur servir à la défense et à l'attaque. Et c'est ainsi que la ligue, couvrant tout l'arrière-pays du Saint-Laurent et devenue de plus en plus puissante par le matériel européen, serait venue à bout, par la destruction ou la migration forcée, des Iroquois de Stadaconé et d'Hochelaga. ${ }^{9}$

Quoi qu'il en soit, la vallée du Saint-Laurent a retrouvé une certaine unité politique; une nouvelle hégémonie favorise les Français du dix-septième siècle: celle de la ligue des Montagnais, Etchemins et Algonquins, ligue qui a l'appui militaire des Micmacs ou Souriquois de l'Acadie ${ }^{10}$ et des Hurons de la lointaine baie Georgienne. Les nations du Saint-Laurent, parce qu'elles sont nomades, vont faciliter la colonisation européenne, et parce qu'elles comptent de plus en plus sur le troc européen, voudront défendre la vallée contre les attaques de ces Iroquois qui vivent en sédentaires au sud du lac Ontario.

\section{LA TOPONYMIE SE DONNE UN NOUVEAU VISAGE}

A cette révolution ethnique correspond une transformation importante de la toponymie.

Malgré le désir qu'en avait de Monts, Chauvin ne s'était pas préoccupé en 1600 de faire de la «descouverture »; aussi, dans les cartes de la Nouvelle-France, ne faut-il s'attendre, en cette fin du seizième siècle, à aucun progrès dans la connaissance géographique. Toutes les nombreuses cartes publiées de 1543 à 1600 en demeurent toujours, pour le dessin des terres, à l'information qu'on avait acquise par les voyages de Cartier et de Roberval: le Cap-Breton, les îles de la Madeleine, la région gaspésienne et ce fleuve qui, à l'ouest d'Hochelaga, se termine en deux branches demeurent, dans la cartographie, ce qu'ils étaient con-

${ }^{9}$ A. G. Bailey, article cité, dans MSRC, 27 (1933) : 97-108.

10 Champlain, op. cit., I: 170. 


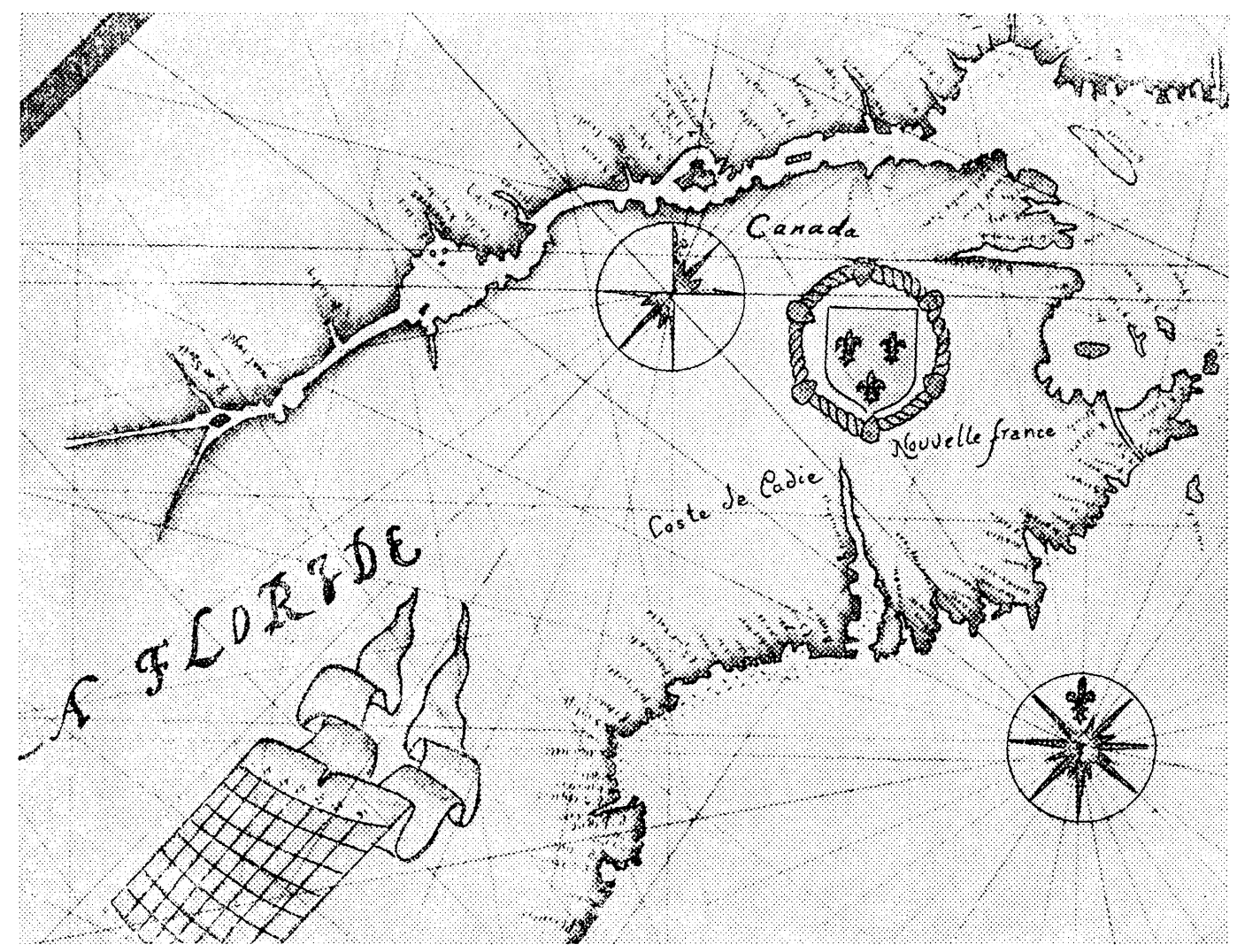

PARTIE LAURENTIENNE DE LA CARTE LEVASSEUR (1601)

Comme du temps de Cartier, la connaissance géographique ne dépasse pas Hochelaga. Notons toutefois que d'importants toponymes font leur première apparition dans la cartographie: île Bonaventure, Gaspé, Québec, Trois-Rivières. 
nus en 1543. Compte tenu du seul dessin géographique, toute carte publiée au cours de ce demi-siècle pourrait en somme se présenter comme une carte de 1543. C'est le cas, par exemple, de cette carte Mercator de $1569^{11}$ : bien qu'elle soit la carte du monde la plus importante du seizième siècle, elle ne marque, pour le Saint-Laurent, aucun progrès. Il en est à peu près de même de la carte Levasseur en 1601. Cette dernière assurément est d'aspect moderne et ce qu'il y a de mieux en cartographie avant Champlain: Terre-Neuve, enfin présentée comme une île d'un seul bloc, se rapproche de sa forme réelle; le golfe est mieux dessiné et le fleuve a des traits plus exacts, mais la péninsule acadienne est encore inexistante et l'on ne connaît de la vallée laurentienne rien de plus que ce qu'en savaient les Français en 1543. Pendant ce demi-siècle, la connaissance géographique demeure stagnante.

Ce que la carte Levasseur présente de neuf en 1601, c'est la transformation toponymique. Les cartes antérieures, s'inspirant des explorations de Cartier et de Roberval, affichaient toujours la même toponymie iroquoise ou celle que les Français avaient imposée de 1534 à 1543. De ces toponymes français plusieurs n'apparaissent plus à la fin du seizième siècle: les Araines, le cap de Lorraine, le cap du Sauvage, la baie SaintLunaire, l'île de l'Assomption avec ses caps Montmorency et Saint-Louis, Charlesbourg-Royal, France-Roy, France-Prime, rivière de Fouez. Des toponymes iroquois, comme Honguedo et Tutonaguy, ont aussi disparu; d'autres survivent, Saguenay, Adgona, Canada, Hochelay et Hochelaga, mais à part Saguenay et Canada ils ne sont plus qu'une réminiscence d'une civilisation évanouie.

11 Carte reproduite dans Trudel, Atlas historique, 23. Elle a exercé une grande influence sur son temps, en particulier sur des cartes importantes comme celles d'Ortelius en 1570, de John Dee en 1580, de Petrus Plaucius en 1592, d'Emery Mollineux en 1592 et de Wytfliet en 1597. La carte Mercator contient, pour la Nouvelle-France, quelques erreurs: le détroit Saint-Pierre a été placé au sud de l'Anticosti; Honguedo est rendu sur le Saguenay. Au point de vue toponymie, cette carte marque progrès, puisque, pour la première fois, le golfe, d'ordinaire appelé Grande baie, porte le nom de Saint-Laurent, ce nom que Cartier avait donné à une petite baie de la rive nord. 
Une autre toponymie est mise en place. Aux toponymes français qui sont des survivances de l'époque de Cartier ${ }^{12}$, Levasseur en ajoute quelques autres qu'il est le premier à inscrire sur une carte: cap Saint-Laurent à l'extrémité du Cap-Breton, île Saint-Jean (maintenant île du Prince-Édouard), îles Ramées qu'il écrit Cames, îles Bonaventure, baie des Morues, Pic qui deviendra Le Bic, Anse-aux-Navires à l'entrée du Saguenay, et Trois-Rivières. Les additions les plus intéressantes viennent de langues indigènes autres que l'iroquois. Déjà avant Levasseur, la toponymie subissait l'influence de ces relations qu'entretenaient les pêcheurs de morue sèche avec les naturels de la côte: en 1558, par exemple, le géographe Homem inscrivait sur le territoire des Micmacs des noms qui seraient d'origine micmacque: macaracade, maracadi, pescagudique, michcomai ${ }^{13}$; c'était là un phénomène tout nouveau et exceptionnel pour l'époque. En 1601, la toponymie indigène prend une place imposante et définitive dans la cartographie: trigety, gaspay et gaspi, natistcoti (notre Anticosti), Uscanimes (les Escoumains), tadoucaq, quebec ${ }^{14}$. Parce qu'elle n'a plus de support réel, la toponymie iroquoise se fait supplanter par la toponymie micmacque, montagnaise ou algonquine.

L'installation définitive de ces toponymes français et indigènes indique que nous entrons dans une époque nouvelle, bien différente de Cartier; et certains d'entre eux (comme île Saint-

$12 \mathrm{De}$ ces survivances de l'époque de Cartier mentionnons (TerreNeuve mise à part) île Bryon, îles Margaux, baie de Chaleur, cap d'Espoir, monts Notre-Dame, Blanc-Sablon, Brest, cap de Rabast, baie St-Laurent, Sept-Isles, île aux Coudres, île d'Orléans, Ste-Croix, lac d'Angoulême, Bastille, rivière St-Malo, Mont-Royal. Quant à fort Henri-Charles (sur le site de Charlesbourg-Royal) et à rivière de Sault (rivière des Outaouais), nous les considérons aussi comme des survivances de l'époque de Cartier.

13 Voir là-dessus, Hoffman, Cabot to Cartier, 188-196.

14 Thevet vers 1550 avait déjà parlé de Naticousti; quant à Tadoussac, il avait paru aussi dans Thevet sous la forme Thadoyzeau; nous le retrouvons écrit Tadouscu sur la carte Hakluyt-Wright de 1599-1600 (c'est la première fois alors qu'il apparaît sur une carte). Pour le gaspay ou gaspi de Levasseur, c'est la première apparition en cartographie; c'est dans une lettre de janvier 1603 que ce toponyme apparaît dans les textes (lettre de Charles de Montmorency, 3 janv. 1603, dans Ramé, Documents inédits, éd. 1867, 19-21). Sur ces questions, voir aussi Hoffman, Cabot to Cartier, 173, 188-196; Ganong, Crucial Maps, IX, dans MSRC, 31 (1937), II : 121 s. 
Jean, Gaspé, Tadoussac, Québec et Trois-Rivières) vont désormais rester associés aux grands événements de notre histoire.

Époque nouvelle aussi, au point de vue de la colonisation. $\mathrm{Au}$ début de 1603, Henri IV travaille à unir les marchands de Rouen et de Saint-Malo pour mieux soutenir Chauvin, titulaire du monopole laurentien; il envoie un commissaire à l'île de Sable pour savoir exactement ce que vaut la tentative de La Roche. A partir de 1603, il s'établit chez les rois de France une tradition désormais constante, et non plus occasionnelle, de politique coloniale.

\section{LA BREVE CARRIËRE AMÉRICAINE D'AYMAR DE CHASTE}

Par suite du décès de Chauvin, le monopole du Saint-Laurent va-t-il revenir tout entier à La Roche qui s'occupe toujours de son île de Sable? En ce début de 1603, La Roche prépare un autre voyage pour ravitailler sa colonie; se fondant sur ses lettres-patentes, il réclame toujours Tadoussac comme partie de son domaine, mais Henri IV ne semble pas convaincu de la solidité des travaux de La Roche ${ }^{15}$; il le laisse de côté et choisit pour succéder à Chauvin celui-là même qui devait présider la rencontre des marchands rouennais et malouins, le commandeur Aymar de Chaste.

Sa carrière européenne est impressionnante: chevalier de Malte, grand-prieur d'Auvergne, commandeur de Limoges, grand-maître de l'Ordre de Saint-Lazare, il est envoyé au Portugal en 1582 pour y remplacer Strozzi qui a péri dans la lutte contre l'Espagne: Aymar de Chaste revient défait, mais héros d'un combat chevaleresque; en 1586, il s'empare de l'île d'Oléron sur les huguenots ; vice-amiral de France et gouverneur de Dieppe en 1589, il fait, comme Chauvin, Pont-Gravé et de Monts, du service pour la cause du protestant Henri IV, service qui n'était pas que militaire puisqu'en 1593 il est créancier du roi pour une somme de 93,000 livres; la guerre terminée, il forme une association de négociants dont fait partie Pont-Gravé; en 1602,

15 Doc. du 21 fév. 1603, dans RHAF, II, 2 (sept. 1948) : 211. 
il se rend en Angleterre comme ambassadeur extraordinaire d'Henri IV ${ }^{16}$. Personnage fort imposant, de Chaste est catholique. Champlain, qui lui doit beaucoup, lui rend un hommage tardif mais généreux: "Bien qu'il eust la teste chargée autant de cheveux gris que d'années, [de Chaste] vouloit encores laisser à la postérité par ceste loüable entreprise, une remarque tres charitable en ce dessein, \& mesmes s'y porter en personne, pour consommer le reste de ses ans au service de Dieu \& de son Roy, en y faisant une demeure arrestée, pour y vivre \& mourir glorieusement. ${ }^{17}$

Malgré l'importance du nouveau titulaire, les marchands de Saint-Malo refusent d'entrer en société avec ceux de Rouen: pour eux, la colonisation est une corvée sans intérêt; mais ils ne cessent de revendiquer la liberté du trafic, en raison de la découverte du Canada qui, disent-ils, a été faite «avecq grande despense par leurs prédécesseurs »: Henri IV leur permet pour cette année 1603 d'équiper un vaisseau qui ira commercer au Canada sous la conduite du capitaine Gilles Eberard du Coulombier; ce vaisseau pourra même faire le voyage en compagnie des navires d'Aymar de Chaste ${ }^{18}$. Le monopole restait sauf.

De Chaste alors constitue une compagnie des seuls marchands de Rouen ${ }^{19}$; pour le représenter en Amérique, il désigne Pont-Gravé, qui avait déjà une longue expérience du SaintLaurent ${ }^{20}$, et il nolise deux navires, la Bonne-Renommée et la Françoise, cette dernière toujours sous les ordres du capitaine Jehan Girot ${ }^{21}$. Un associé de Pont-Gravé, le Malouin Jean Sarcel

16 E. Gosselin, Nouvelles glanes, 20; Régis Roy, M. de Chaste, dans BRH, 27 (1921) : 214s.; Biggar, The Early Trading Companies, 46.

17 Champlain, Oeuvres (éd. Biggar), III : 312s. Champlain ne dit pas un mot d'Aymar de Chaste dans l'édition de 1603 (qui raconte un voyage pourtant organisé par de Chaste), ni dans l'édition de 1613: il attend 1632; nous ignorons pourquoi ce long retard de 29 ans.

18 Lettre du roi, 13 mars 1603, dans Ramé, op. cit., 23-25; AN, série E, vol. $5 \mathrm{~A}$, fol. 248.

19 Champlain, op. cit., III: 313. Parmi les associés, mentionnons Pont-Gravé, Thomas Legendre, Louis de Petigas de La Guérinière (doc. dans les frères Bréard, Doc. relatifs à la marine normande, 92).

20 Champlain, op. cit., III : 314.

21 Documents de février et mars 1603, dans les Bréard, op. cit., 99-101; Champlain, op. cit., I: 100. 
de Prevert, se joint à l'expédition, mais sur son propre navire ${ }^{22}$. Pont-Gravé quitte Honfleur le 15 mars 1603, dans le temps même que La Roche envoyait un dernier navire à l'île de Sable.

Aymar de Chaste ne devait pas voir revenir sa flotte: deux mois plus tard, avant même que Pont-Gravé n'eût atteint Tadoussac, de Chaste décédait ${ }^{23}$. Entre le moment où Henri IV l'avait nommé pour réunir Rouennais et Malouins dans l'entreprise de Chauvin et celui de sa mort, il avait eu tout juste le temps d'hériter du monopole et de mettre en mer l'expédition de Pont-Gravé. Rôle bref mais important. L'histoire retiendra de lui qu'il est l'organisateur du célèbre voyage de 1603, mais la géographie canadienne n'a perpétué son nom que par un cap, le cap de Chaste, déformé d'ailleurs en Cap-Chat ${ }^{24}$.

\section{UN OBSERVATEUR, SAMUEL DE CHAMPLAIN}

Parmi ceux qui s'embarquent en ce mois de mars 1603, nous trouvons deux sauvages que Pont-Gravé avait amenés en France lors d'un précédent voyage; signalons encore Pierre Chauvin de La Pierre, parent de feu Chauvin de Tonnetuit ${ }^{25}$; et un nouveau personnage jusque-là inconnu, Samuel de Champlain.

Il s'embarque, non à titre de lieutenant ainsi qu'on l'a déjà écrit ${ }^{26}$, mais en simple observateur comme l'était de Monts en 1600. Selon sa propre déclaration, il avait été invité par Aymar de Chaste à «voir ce pays, \& ce que les entrepreneurs y feroient»; de Chaste obtint pour Champlain la permission nécessaire, et Pont-Gravé reçut l'ordre de le « recevoir en son vaisseau » et de lui «faire voir \& recognoistre tout ce qui se pourroit en ces lieux ${ }^{27}$. Âgé d'une trentaine d'années, Champlain cherchait sa voie.

22 Champlain, op. cit., I: 169,180 s.

23 Il serait mort le 13 mai 1603 (N.-E. Dionne, Champlain, I: 73; Roy, $M$. de Chaste, dans BRH, 27, 1921: 214s.).

24 Ce toponyme apparaît pour la première fois sur une carte de 1612 (carte de Champlain), écrit C. de chate.

25 Champlain, op. cit., I : 98; les Bréard, op. cit., 99s. et n. 3.

26 Régis Roy, M. de Chaste, dans BRH, 27 (1921) : 214.

27 Champlain, op. cit., III: $315 \mathrm{~s}$. 
Qu'avait-il fait avant 1603? Quel est ce mystère qui l'entoure jusque-là, comme Colomb, Cabot, Verrazano et Cartier ? Son acte de naissance est introuvable, un incendie ayant détruit dans sa ville natale, Brouage, les registres antérieurs à $1690^{28}$. On calcule généralement qu'il est né vers 1570 , sinon en 1567 . On a voulu voir en lui tantôt un fils de pauvres pêcheurs, tantôt un bâtard d'une grande famille ${ }^{29}$. Halluciné par ce mystère, Florian de La Horbe a tenté de le déchirer en dénouant l'énigme à la façon d'un Alexandre Dumas: selon La Horbe, Champlain ne serait que ce Guy Eder de La Fontanelle, l'un des plus terribles soudards des guerres de religion, qui condamné à être rompu vif aurait échappé au châtiment pour des raisons politiques et serait réapparu tout pacifié, tout rangé, sous le nom de Samuel de Champlain; dès 1603, il n'était déjà plus reconnaissable par ses adversaires ${ }^{30}$. En vain cherchons-nous dans cette thèse une preuve sérieuse qui nous fasse au moins douter de l'histoire traditionnelle: le livre de La Horbe n'est qu'un fort mauvais roman policier et nous n'en faisons ici mention que pour le bénéfice des collectionneurs d'œuvres cocasses.

D'après le contrat de mariage de 1610 , Champlain serait fils «de feu Anthoine de Champlain, vivant capitaine de la Marine, et de dame Marguerite Le Roy » ${ }^{31}$; nous n'en sommes guère plus avancés: il était facile de donner du de et des qualités à ses parents dans un acte par-devant notaire, mais Champlain, lui-même capitaine de la Marine, a pu marcher dans la carrière $\mathrm{du}$ père. Nous ne savons rien d'autre sur ses parents et il reste encore à élucider le mystère de cet «oncle Provençal»

${ }^{28}$ A.-L. Leymarie, Inédit sur le fondateur de Québec, dans Nova Francia, I (1925) : 80-85.

29 Hypothèses relevées par H. Deschamps, dans l'introduction aux Voyages de Samuel Champlain, 4, n. 5.

30 Florian de La Horbe, L'incroyable secret de Champlain. Voir aussi là-dessus, une recension de L. Groulx, dans RHAF, XIII, 2 (sept. 1959) : 289-292.

31 Contrat du 27 déc. 1610 , reproduit dans Champlain, op. cit., II: 315-317; et dans Dionne, Champlain, I: 399-403. 
qui joue un rôle important dans les débuts de la carrière de Champlain ${ }^{32}$.

Nous ne savons pas si Champlain fut baptisé catholique ou protestant: son prénom biblique qui en Saintonge n'était guère donné que dans les familles protestantes, et la ville huguenote qu'était alors Brouage, rendent probable cette naissance protestante de Champlain ${ }^{33}$. Le lutte qu'il a faite contre la Ligue catholique ne prouve rien, car l'opposition à la Ligue était le fait de monarchistes catholiques ou protestants; ne prouve rien non plus son choix d'une épouse dans la société protestante. S'il est né protestant, Champlain est passé très tôt au catholicisme, étant dans le cas du jésuite Paul Le Jeune, né huguenot et devenu catholique à sa majorité ${ }^{34}$. Quoi qu'il en soit, lorqu'il commence en 1603 sa carrière canadienne, il est catholique: la doctrine qu'il explique alors aux sauvages de Tadoussac est une doctrine catholique ${ }^{35}$.

Etait-il noble? Le peu que nous connaissons de sa famille ne nous éclaire pas là-dessus. Ni le nom qu'il porte: l'édition de 1603 donne Samuel Champlain et la dédicace à l'amiral Montmorency est signée $S$. Champlain, alors que dans le privilège de cette même édition on écrit Sieur de Champlain, tout comme dans le contrat de mariage en 1610 , et dans les éditions de 1613, de 1619 et de 1632. Toutefois, la particule ne prouve rien, la noblesse ne s'établit que par filiation noble ou par les lettres de noblesse. Faute de ces dernières (qu'elles n'aient pas existé ou qu'elles se soient perdues), il faut recourir, avec beaucoup de prudence, aux titres qu'il prend ou se laisse donner. Dans le contrat de mariage de 1610, on le qualifie de noble homme: c'est une qualité qu'on donnait aux gens importants qui n'étaient pas nobles. Or, dans un acte notarié de 1615 , Champlain est qualifié d'écuyer: seuls les nobles avaient droit à ce titre, bien qu'en

32 Marcel Delafosse, L'oncle de Champlain, dans RHAF, XII, 2 (sept. $1958)$ : 208-216; L.-A. Vigneras, Encore le capitaine provençal, dans RHAF, XIII, 4 (mars 1960 ) : 544-549.

33 H. Deschamps, Les voyages de Samuel Champlain, 4, n. 3, et 5. 34 RJ, V: 275.

35 Champlain, op. cit., I: 115s. Ce qu'il a expliqué aux sauvages de Tadoussac, il l'imprime dès 1603 . 
pratique plus d'un bourgeois se laissât donner de l'écuyer sans broncher. Puis, dans un engagement de 1617, on lui donne du noble homme, comme aussi dans un document de 1621; mais, dans un acte notarié de 1625 et, en 1626, lors de l'insinuation de cet acte, Champlain retrouve son titre d'écuyer; bien plus, dans la liste officielle des Cent-Associés de 1627, dressée avant que le roi remette à la Compagnie douze lettres d'anoblissement pour des Associés non nobles, on qualifie encore Champlain d'écuyer ${ }^{36}$. Si l'on pouvait ne pas tenir compte des documents de 1617 et de 1621, on pourrait conclure que Champlain, noble homme en 1610, a pu accéder à la noblesse avant 1615; en ce cas, la promotion se situerait en 1612 , lorsque Champlain devient lieutenant d'un vice-roi: on voit mal un Bourbon de Condé se faire représenter en Nouvelle-France par un simple roturier qui reçoit des pouvoirs étendus que seul un noble peut en principe exercer. C'est là une hypothèse: il faudra pour l'étayer plus solidement la découverte de nouveaux documents.

De ses débuts nous savons peu de chose de certain. Il a pu pratiquer un art nécessaire au géographe, celui de peintre ou dessinateur; un factum des environs de 1613, rédigé par les marchands malouins, note à propos du Champlain de 1603: « [A ce voyage] ayant été seulement comme passager, sa profession de peintre le conviant, avecques le lucre, de veoyr ledicts pays ${ }^{37}$. En fait, Champlain fut un excellent dessinateur: les dessins de ce Brief discours qu'on lui attribue (et qui semblent originaux alors que le texte n'est qu'une copie) sont très heureux, d'une belle main; les cartes qu'il dessinera de la Nouvelle-France, surtout celle de 1632, sont d'une magnifique facture. Il a dû commencer jeune à naviguer, puisqu'il déclare à la reine en 1613 qu'il a été attiré par l'art de la navigation dès son «bas aage » ${ }^{38}$. Il affirme en 1632 qu'il a servi contre la Ligue dans

36 Acte du 18 mars 1615, dans Gosselin, Nouvelles glanes, 45. Document de 1625, cité par Leymarie, dans Nova Francia, I (1925) : 83-85. AN, Colonies, C 11 A, 1: 114v. On s'est demandé si Champlain n'aurait pas été anobli par sa participation à la Compagnie des Cent-Associés; mais nulle part il n'est établi que Champlain était des 12 roturiers que le roi avait promis d'anoblir pour les récompenser. D'ailleurs, qualifié d'écuyer dans la liste officielle de 1627, Champlain portait déjà ce titre en 1615. 37 Cité par Deschamps, Les voyages de Samuel Champlain, 44, n. 2. 38 Champlain, op. cit., I: 209. 
l'armée de Henri IV jusqu'en 1598, avec le titre de maréchal des logis; quand les troupes espagnoles, entretenues par Philippe II en Bretagne, quittent Port-Blavet (aujourd'hui Port-Louis, dans le Morbihan), Champlain s'embarque avec elles; et d'Espagne, à ce qu'il affirme au roi en 1630 et répète en 1632 , il poursuit jusqu'aux Indes occidentales un voyage qui le retient deux ans et demi. On constate sa présence à Cadix en juillet 1601, puis il rentre en France: à la Cour, il jouit d'une pension dont l'honore le roi. C'est alors qu'il entre en relation avec le commandeur de Chaste, celui-ci l'invite à suivre Pont-Gravé. ${ }^{39}$

Dans un mémoire au roi vers 1630 et dans une œuvre de 1632, Champlain affirme qu'il a voyagé deux ans et demi dans les Indes occidentales: on ne voit pas pourquoi on devrait rejeter cette déclaration qui vient d'un homme à la fin de sa carrière. Ce qui est venu compliquer le problème, c'est une œuvre que Champlain n'a jamais publiée mais qu'on lui attribue: Brief discours des choses plus remarquables que Samuel Champlain de Brouage a reconnues aux Indes occidentales ${ }^{40}$. Claude de Bonnault et Jean Bruchési ont été les premiers à mettre en doute l'authenticité de cet écrit et, partant, la sincérité de Champlain ${ }^{41}$. Une étude récente, celle de L.-A. Vigneras, repense le problème en retenant quelques hypothèses: Vigneras constate que le Brief discours donne parfois un itinéraire incompatible avec celui de l'armada de Coloma, que Champlain est censé avoir accompagnée; il faut en outre, continue Vigneras, «faire les plus grandes réserves sur les prétendus voyages à Mexico, Porto-Bello et Carthagène »; et comment expliquer les erreurs grossières que contient le Brief discours ? Selon Vigneras, Champlain n'aurait pas fait son voyage ou tout son voyage avec la flotte de Coloma; ou il aurait rédigé le Brief discours

39 Champlain, op. cit., III: 314s.; cession de la donation de 1601 par l'acte du 29 déc. 1625 , reproduit par Leymarie, dans Nova Francia, I (1925) : 83-85; mémoire publié par Deschamps dans Les voyages de Samuel Champlain, 354. Dans le Brief discours, on précise que le départ s'est fait à bord du St-Julien en compagnie de l'oncle, le capitaine Provençal (Champlain, op. cit., I: $3-5$ ).

40 Reproduit dans l'édition Biggar des Oeuvres de Champlain, I: 3-80.

41 Claude de Bonnault, cité par Deschamps, Les voyages de Samuel Champlain, 48, n. 2; Bruchési, Champlain a-t-il menti? dans les Cahiers des Dix, XV (1950) : 39-53. 
d'après des renseignements recueillis en Espagne, sinon d'après les papiers du fameux «oncle provençal»; ou bien il n'aurait raconté son voyage qu'à une époque où sa mémoire n'était plus du tout fidèle. ${ }^{42}$

Pour être juste à l'égard de Champlain, il faut d'abord retenir qu'il n'a jamais publié le Brief discours: ou bien cette œuvre n'est pas de lui ou, si elle l'est, il ne l'a pas jugée digne de paraître; on ne doit pas la compter avec les autres œuvres de Champlain. Autre remarque non moins importante: ce qu'on a publié sous le nom de Champlain, et seulement à partir de 1859 , ce n'est pas l'original mais une copie ${ }^{43}$; dans quelle mesure cette copie est-elle fidèle ? La même aventure est arrivée à la relation de Verrazano que l'on ne connut longtemps que par une copie tronquée et parsemée d'erreurs: il a fallu la découverte du manuscrit Cellere pour que les historiens rendent enfin à Verrazano son juste mérite. Jusqu'à ce que l'original soit retrouvé, on n'a pas le droit de verser le Brief discours au dossier de Champlain.

Ce Champlain jusque-là énigmatique, navigateur et peintre, protégé du roi et du commandeur Aymar de Chaste, s'embarque comme simple passager. Il n'exerce encore aucune fonction précise; il n'est pas encore capitaine de la Marine: lorsqu'il publiera sa relation à son retour, aucun titre ne suit son nom. Était-il géographe $d u$ roi, comme le saluera Lescarbot dans un sonnet de 1607 ? ${ }^{44}$ Nulle part Champlain ne porte ce titre et personne d'autre que Lescarbot ne le lui donne; rien n'établit que Champlain, tout en agissant en géographe, ait occupé le

${ }^{42}$ L.-A. Vigneras, Le voyage de Samuel Champlain aux Indes occidentales, dans RHAF, XI, 2 (sept. 1957) : 163-200. Voir aussi les études citées plus haut, note 32 de ce chapitre.

43 Voir Vigneras, étude citée. Ce Brief discours a été publié pour la première fois, et ce en anglais, en 1859 par la Hakluyt Society d'après un manuscrit conservé à la John Carter Brown Library de Providence (R.I.) et tiré d'une bibliothèque privée de Dieppe. Ce manuscrit servit aussi à l'édition Laverdière de 1870 et à celle de la Champlain Society en 1922. On a repéré deux autres manuscrits, l'un à Turin et l'autre à Bologne, mais aucun d'eux n'est encore l'original.

44 Sonnet de Lescarbot, rédigé en 1607 à Canseau (Les muses de la Nouvelle-France, dans Histoire de la Nouvelle-France, éd. Tross, III: 52; voir aussi l'édition Grant \& Biggar, III: 494); Histoire de la NouvelleFrance, éd. Tross, III: 596. 
poste officiel de géographe du roi. En 1603, il s'embarque en simple observateur et sa présence en ce voyage serait passée inaperçue s'il n'avait publié sa relation; il est d'ailleurs le seul à nous raconter ce voyage de 1603 .

\section{LA «TABAGIE » DE TADOUSSAC}

Le 7 mai 1603, l'expédition d'Aymar de Chaste, conduite par Pont-Gravé, était en vue de Terre-Neuve; le 20, elle arrive à l'île d'Anticosti et, le lendemain après avoir aperçu "Gachepé », elle entre dans le fleuve Saint-Laurent qu'elle remonte jusqu'au Bic pour de là traverser à Tadoussac où elle arrive enfin le 26 mai ${ }^{45}$.

A une lieue de là, sur la pointe Saint-Mathieu (aujourd'hui Pointe-aux-Alouettes), une centaine de sauvages faisaient «tabagie » (c'est-à-dire festin) sous la direction du chef Anadabijou. L'un des indigènes que Pont-Gravé ramenait de France, leur raconte «la bonne reception que leur avoit fait le Roy, \& le bon traictement qu'ils avoient receu en France », les «beaux Chasteaux, Palais, maisons \& peuples qu'ils avoient veus, \& nostre façon de vivre »; le roi, assure cet indigène, "leur vouloit $\mathrm{du}$ bien, \& desiroit peupler leur terre, \& faire paix avec leurs ennemis (qui sont les Irocois) ou leur envoyer des forces pour les vaincre ». Anadabijou se déclare fort enchanté d'avoir le roi «pour grand amy » et fort aise qu'il peuple le pays et fasse la guerre aux ennemis. Puis on danse pour fêter une récente victoire sur les Iroquois et tous les sauvages (Algonquins, Etchemins et Montagnais) se transportent à Tadoussac même, au nombre de mille, avec leurs canots d'écorce de bouleau chargés de fourrures ${ }^{46}$.

Là, nouvelle tabagie. Présidés par leur chef Tessouat (ou Besouat), les Algonquins font danser nues leurs Algonquines, «femmes bien formées, remplies \& potelées, de couleur basanée »: les Etchemins et les Montagnais apportent des présents; on se livre ensuite à des concours de vitesse, encore couronnés

45 Champlain, Oeuvres (éd. Biggar), I : 91-96.

46 Ibid., I: 98-106. 
de présents ${ }^{47}$. Pour Pont-Gravé, habitué de la traite de Tadoussac, le spectacle n'était pas nouveau; il l'était pour Champlain.

Pendant cette traite, du 26 mai au 18 juin, Champlain étudie à loisir les indigènes. Peuples d'une humeur assez joyeuse, ils parlent fort posément, «promettent assez \& tiennent peu »; sont grands menteurs et «la pluspart gens qui n'ont point de loy »; ils croient cependant en un Dieu qui a créé toutes choses, mais sans aucune cérémonie pour le prier, et en l'immortalité de l'âme. Les songes qu'ils font, ils les croient véritables. Ils s'habillent de peaux en laissant une partie du corps à découvert; ils s'enveloppent de bonnes fourrures l'hiver et vont sur la neige en s'attachant aux pieds «une maniere de raquette». A 14 ou 15 ans, l'Indienne s'associe à qui elle veut, puis, après cinq ou six ans, elle prend pour mari celui qui lui plaît et lui demeure fidèle. On les inhume avec tout ce qu'ils ont: chaudrons, fourrures, haches, arcs et flèches.

Champlain leur sert un cours de religion, dont la matière est en tous points conforme à la doctrine catholique. Et il espère qu'on puisse civiliser un jour ces indigènes: "je tiens que qui leur monstreroit à vivre \& enseigner le labourage des terres, \& autres choses, ils l'apprendroient fort bien; car je vous asseure qu'il s'en trouve assez qui ont bon jugement, \& respondant assez bien à propos sur ce que l'on leur pourroit demander. ${ }^{48}$

Le 11 juin, Champlain profita de ses loisirs pour remonter le Saguenay sur une distance de douze ou quinze lieues, visitant la partie qui ressemble plus à un fjord qu'à une rivière: "profondeur incroyable 》, écrit-il, «montaignes de rochers 》 où il n'y a pas « une lieue de terre plaine tant d'un costé que d'autre », « vrais deserts inhabitables d'animaux, \& d'oyseaux 》. Les indigènes lui décrivent un «torrent d'eau d'une grande impetuosité » d'où coule cette rivière, torrent précédé de 18 saults après quoi on parvient à un grand lac qu'alimentent des rivières: c'est déjà tout le plan hydrographique du royaume du Saguenay, avec

47 Ibid., I: $107-109$.

18 Ibid., I : 110-120. 
sa chute principale (Arvida-Shipshaw) et le lac Saint-Jean. $\mathrm{Au}$ bout de ces rivières qui viennent se déverser dans le lac, lui disent les indigènes, il y a d'autres lacs; par ces voies d'eau descendent des nations du nord qui font avec les Montagnais le troc des peaux de castor et de martre en échange de ce que les Français donnent aux Montagnais; ces nations du nord, selon les indigènes, "voyent une mer qui est salée ». Au lieu de conclure tout de suite, comme les voyageurs intéressés, qu'il s'agit de la mer d'Asie, Champlain écrit avec une assurance qui nous étonne: «c'est quelque gouffre de ceste mer qui desgorge par la partie du Nort dans les terres, \& de verité il ne peut estre autre chose ${ }^{49}$. Bien que la baie d'Hudson ne soit pas encore découverte, Champlain la devine ici, pour ainsi dire; certes, une carte de Wytfliet en 1597 dessinait au nord-ouest du Saguenay, à peu près où se trouve la baie d'Hudson, ce lac des Conibas ${ }^{50}$ qui correspond exactement à ce «gouffre» de la mer du Nord. Champlain connaissait sans doute Wytfliet; en tout cas, il a su résister à la tentation de découvrir la mer d'Asie au bout du Saguenay.

Probablement le premier explorateur à pénétrer aussi avant dans le Saguenay, Champlain note donc deux détails de grande importance pour la connaissance de cette partie du Nouveau Monde: d'une part, l'existence d'une mer salée qui n'est pas la mer d'Asie; d'autre part, une route commerciale, établie depuis assez longtemps, semble-t-il: celle que suivent les nations du Nord pour descendre traiter avec les Montagnais.

\section{CHAMPLAIN REMONTE LE SAINT-LAURENT}

Tabagie et traite terminées, Pont-Gravé entreprend le 18 juin la montée de ce fleuve que Champlain appelle encore, comme le faisait Cartier, rivière de Canada. Pont-Gravé veut se rendre en barque jusqu'au sault Saint-Louis: il n'avait jamais été plus loin que les Trois-Rivières, bien qu'en 1600 il eût voulu pousser plus en amont. Champlain l'accompagne. En ce voyage

49 Ibid., I: 121-124.

50 Voir cette carte de Wytfliet dans Trudel, Atlas historique $d u$ Canada français, 27. 
de 1603 sur le Saint-Laurent, Champlain ne découvre pas; ce qui est nouveau pour lui ne l'est pas pour les Français de son temps: depuis un demi-siècle, la cartographie représente assez exactement le fleuve jusqu'à Hochelaga; la carte de Levasseur en 1601 portait déjà les toponymes Tadoussac, Québec et TroisRivières.

Île aux Lièvres, île aux Coudres, île d'Orléans: lieux connus et ainsi appelés depuis Cartier. Au bout de l'île d'Orléans, comme Cartier, Champlain admire « un torrent d'eau qui desbordoit de dessus une grande montaigne $\gg^{51}$; il lui donne, à une date qu'on ne peut préciser, le nom de Montmorency ${ }^{52}$. Le 22 juin, on jette l'ancre «à Quebec qui est un destroict de ladicte riviere de Canadas », toponyme indigène qui signifierait resserrement ${ }^{53}$ et dont la carte Levasseur avait consacré l'usage deux ans plus tôt. Ici Champlain s'en tient à des généralités géographiques: si les terres étaient cultivées, elles seraient bonnes comme celles de France; comme Cartier, il voit « des diamans dans des rochers d'ardoise, qui sont meilleurs que ceux d'Alançon». Persuadé que Cartier s'était établi en amont, il ne s'amuse à aucun rappel historique; il ne songe non plus à aucun projet d'avenir: le site l'impressionne, c'est tout. D'ailleurs, l'on s'arrête à peine: Pont-Gravé est dans des lieux qui lui sont familiers et il repart en amont dès le lendemain.

En route, Champlain se laisse tromper et situe l'établissement de Cartier à quinze lieues en haut de Québec, dans une rivière qui depuis a gardé le nom de Sainte-Croix. Il signale des rapides qui recevront un jour le nom de rapide Richelieu (entre Deschambault et Lotbinière); la rivière Batiscan «par où quelques-fois les Algonquins viennent » et dont le nom semble en usage avant 1603; un peu au-delà de l'embouchure de cette rivière, une île Saint-Eloi que l'on semble connaître sous ce nom

51 Champlain, op. cit., I: 125-128.

52 Il se peut que le nom ait été donné dès 1603 : le récit du voyage de 1603 est dédié à l'amiral Charles de Montmorency, duc d'Ampville, mais la carte faite cette année-là par Champlain n'a pas été retrouvée. La première carte de Champlain dans laquelle on trouve la chute Montmorency est la carte des environs de Québec, publiée dans les Voyages de 1613.

53 Champlain, op. cit., I: 129 et n. 2. 
avant l'arrivée de Champlain; puis les trois Rivières (lieu connu sous ce nom depuis au moins la carte de Levasseur en 1601), « où il commence d'y avoir temperature de temps, quelque peu dissemblable à celuy de saincte Croix ». C'est ici que le futur colonisateur commence à se révéler: une île «qui regarde le passage » du fleuve et commande aux autres îles, retient son attention et il écrit: «Ce seroit à mon jugement un lieu propre pour habiter, \& pourroit-on le fortifier promptement, car sa situation est forte de soy, \& proche d'un grand lac». Pour la première fois depuis son arrivée en Amérique du Nord, Champlain parle d'habitation, mais cette habitation est reliée, dans son esprit, au commerce des fourrures: en remontant la rivière (aujourd'hui Saint-Maurice), on peut par des portages parvenir au lac d'où le Saguenay tire sa source (lac Saint-Jean) et de là à Tadoussac; une habitation aux Trois-Rivières serait « un bien pour la liberté de quelques nations qui n'osent venir par là, à cause desdits Irocois, leurs ennemis, qui tiennent toute ladite rivière de Canadas bordée: mais estant habité, on pourroit rendre lesdits Irocois \& autres Sauvages amis, où à tout le moins sous faveur de ladite habitation, lesdits Sauvages viendroient librement sans crainte \& danger ${ }^{54}$. Les circonstances feront que Champlain installera d'abord cette habitation au détroit de Québec; vingt-six ans après, il fera établir par Laviolette le fort des Trois-Rivières.

Un voyage sur le Saint-Maurice dut s'arrêter à une lieue, devant « un sault d'eau fort estroit » et l'on reprit la montée du fleuve. Le 29 juin, fête de saint Pierre, on entre dans le lac appelé jusque-là Angoulême et qui, sur l'initiative de Champlain, deviendra le lac Saint-Pierre ${ }^{55}$. Champlain remarque des rivières qu'il ne nomme pas, trente petites îles à la tête du lac; et il passe dans la rivière des «Irocois 》 (aujourd'hui rivière Richelieu) qu'il veut examiner, car c'est par là que les Iroquois envahissent le Saint-Laurent. Des Montagnais qui allaient leur

54 Ibid., I: 130-138.

55 Dans sa relation de 1603 , Champlain ne dit pas qu'il a donné à ce lac le nom de Saint-Pierre; il écrit seulement qu'il y est entré le 29 juin; ce nom, Saint-Pierre, a dû apparaître pour la première fois sur la carte de 1603 qu'on n'a pas retrouvée; il est, en tout cas, sur la carte de 1611. 
faire la guerre, avaient dressé une palissade depuis le fleuve jusqu'à la rivière pour protéger leurs canots. Sur une barque, puis sur un esquif, Champlain remonte la rivière, mais les rapides l'empêchent de dépasser le présent village de Saint-Ours. Les sauvages lui donnent une bonne description du pays en amont: des rapides de Chambly, la rivière qui conduit parmi des îles du lac Champlain au lac George «au bout duquel sont cabannez les Irocois»; une autre rivière (nous reconnaissons ici l'Hudson) qui a son embouchure à «quelque cent ou cent quarante lieues » sur le littoral atlantique, qu'on appelle encore la « coste de la Floride ${ }^{56}$.

Enfin, le 3 juillet 1603, « avec vent en poupe », la barque de Pont-Gravé arrive en vue du sault Saint-Louis ${ }^{57}$ : ce pays était nouveau pour lui comme pour Champlain. « Nous appareillasmes aussi tost nostre esquif, que l'on avoit fait faire expres pour passer ledit sault ». Pont-Gravé et Champlain y prennent place, quelques guides indiens s'embarquent dans un canot; on parvient ainsi, non sans peine, au pied des rapides: «jamais je ne veis un torrent d'eau desborder avec une telle impetuosité [...] il s'y faict un esbouillonnement estrange de la force \& roideur que va l'eau en traversant ledit sault ». L'obstacle est de taille: "de porter bateaux, c'est chose laquelle ne se peut faire en si bref temps », mais on peut aller au-delà avec des canots et « voir tout ce qui se peut, bon ou mauvais, dans un an ou deux ${ }^{58}$.

\section{DE NOUVEAU, LE MYSTERE DE L'HORIZON OCCIDENTAL}

Il fallut, pour lors, se contenter de questionner les sauvages. Cartier s'était trouvé là en 1535 puis en 1541 , mais sans interprètes; en 1603, des sauvages que l'on comprend vont donner

${ }^{56}$ Champlain, op. cit., I: 138-144, 178. Les mesures approximatives que donne Champlain, sans avoir vu le pays, ne sont pas tellement loin de la réalité: au lac Champlain, qui a 130 milles il donne 40 ou 50 lieues, c'est-à-dire de 120 à 150 milles; il situe l'embouchure de l'Hudson à 100 ou 140 lieues: cette embouchure est à 137 lieues.

57 Ce sault ne portait pas encore ce nom: Champlain écrit seulement: «premier sault » de la rivière de Canada (ibid., 1: 85). Ce toponyme, premier sault, était sur les cartes du seizième siècle.

58 Ibid., I : 144-153. 
des pays d'en haut le premier aperçu qui corresponde clairement à la réalité.

Au-delà des rapides, expliquent-ils, un premier lac où vient se jeter « une rivière qui va en la demeure des Algoumequins », établis à 60 lieues: c'est la rivière des Outaouais, où l'on trouve à 65 lieues en amont de l'embouchure le pays des Algonquins; ce premier lac, où vient se jeter cette rivière, est par d'autres saults séparé d'un autre lac: nous reconnaissons le lac SaintFrançois; encore des saults et l'on rencontre un lac, long de 80 ou 150 lieues: voilà notre lac Ontario, qui mesure environ 65 lieues; en haut de ce dernier lac, un sault «quelque peu élevé » (première allusion, en notre littérature, à la chute Niagara) précède un second grand lac, d'environ 60 lieues: c'est le lac Érié, long de 83 lieues; en amont, un détroit, puis un autre grand lac, dont l'eau est mauvaise: le lac Huron. Les guides n'en ont pas vu la fin, parce qu'ils ne sont pas allés plus outre, «d'autant qu'il est si grand, qu'ils ne se hazarderont pas de se mettre au large ». Champlain comprit que ce lac à eau non potable et dont on ne connaissait pas le bout, serait la «mer du Su» ou mer d'Asie ${ }^{59}$.

Sur la route du retour, on poursuit l'enquête auprès d'Algonquins qui avaient voyagé. Elle apporte des précisions supplémentaires: le dernier grand lac (celui des Hurons) est au nord-ouest; sur la rive nord du premier grand lac (le lac Ontario), où l'eau ne gèle point, vient se déverser une rivière par où descendent les Algonquins: ce serait la rivière Trent qui par des portages communique avec les lacs Rice, Simcoe et la baie Georgienne. Du côté sud de ce premier grand lac, une rivière vient des Iroquois (rivière Oswego ou rivière Black) par où Iroquois et Algonquins se font la guerre. Le pays des Iroquois est fort fertile, «il y a quantité de bled d'Inde » et autres fruits que n'ont pas les Algonquins; une nation appelée les «bons Irocois » (ce seraient les Hurons, apparentés aux Iroquois), vient chez les Algonquins leur acheter des marchandises françaises; selon ces «bons Irocois», il y aurait au nord une mine de franc

59 Ibid., I: 153-160. 
cuivre. Champlain tient compte de la divergence des témoignages (eau salée ou pas, distances plus ou moins longues) et il en déduit que la grande nappe d'eau dont les sauvages n'ont pas vu la fin, ne peut être que «la mer du $\mathrm{Su}$, le Soleil se couchant où ils disent ${ }^{60}$.

Même si les Français de 1603 n'ont pas plus que Cartier le loisir de dépasser l'obstacle du sault Saint-Louis, la connaissance géographique fait ici un progrès immense, progrès qu'elle aurait fait dès 1535 si Cartier avait eu ses interprètes. Tout le réseau hydrologique du haut Saint-Laurent se dessine avec des mesures qui sont assez près de la réalité: immédiatement en amont du sault Saint-Louis, le fleuve se grossit d'un affluent qui vient du pays des Algonquins; en remontant le fleuve, on trouve le lac Saint-François, le lac Ontario dans lequel se déversent une rivière par où on atteint le pays des Algonquins et une autre qui vient du pays des Iroquois; plus haut, on a la chute Niagara, la rivière de Détroit, le lac des Hurons qui s'étend au nordouest. Sur ce dernier point, cependant, l'équivoque reste entière: ce lac n'est-il qu'un lac ou la mer d'Asie ?

De plus, on découvre en haut de l'ancien Hochelaga une grande voie commerciale: les marchandises françaises que les Algonquins viennent troquer dans le Saint-Laurent, passent du haut de la rivière des Outaouais dans le pays des «bons Irocois », c'est-à-dire chez les Hurons. Le seizième siècle vient à peine de finir et déjà les articles européens, articles de métal surtout, parviennent aux indigènes de l'intérieur, à quelque 1,500 milles $\mathrm{du}$ littoral atlantique.

\section{UNE NOUVELLE SÉDUCTION, L'ACADIE}

Revenus à Tadoussac le 11 juillet, Pont-Gravé et Champlain se rembarquent aussitôt pour Gaspé où l'on séjourne du 15 au 19, sans doute pour faire provision de poissons. Ces quelques jours de relâche permettent à Champlain de prendre un aperçu général de la région: baie de Gaspé, rivière qui s'y déverse, baie des «Molues», île Percée, île de «Bonne adventure», tous

60 Ibid., I : 158-165. 
lieux « où il se faict la pesche du poisson sec \& verd». Des sauvages il tire des renseignements sur le pays qu'il ne peut voir: la baie de Chaleur, retraite du «monstre espouvantable », le Gougou; par un affluent de cette baie on peut se rendre à un lac où l'on trouve «dans la terre, environ un pied ou pied \& demy une maniere de metail qui ressemble à de l'argent»; cet affluent permet aussi d'atteindre la rivière Matane, qui va se déverser dans le fleuve. A Champlain on parle de la rivière des Souricois (affluent de la baie de Fundy) où, guidé par le sauvage Secoudon, de la rivière Saint-Jean, le Malouin Sarcel de Prevert est allé à la découverte d'une mine de cuivre "sur le bord de la mer du costé du Su », probablement à Cap d'Or, à l'entrée du Bassin des Mines ${ }^{61}$; on lui parle encore de l'île Saint-Laurent (notre Cap-Breton) où hivernent les Souricois ou Micmacs; de la côte d'Acadie qu'il faut longer pour entrer dans une baie «qui vient joindre ladicte mine de cuivre» (la baie de Fundy); d'une rivière (la rivière Saint-Jean) qui se déverse dans cette baie et par où les sauvages d'Acadie vont faire la guerre aux Iroquois. ${ }^{62}$

Riche en promesses minières que cette Acadie, selon Champlain. Une haute montagne qui avance quelque peu sur la mer et qui est «fort reluisante au Soleil, où il y a quantité de verd de gris qui procede de ladite mine de cuivre » et d'où tombent des morceaux de cuivre; plus bas, vers la côte d'Acadie, une «montaigne qui est d'une peinture noire, dequoy se peignent les Sauvages »; puis, une île où l'on trouve « une maniere de metail » qui ne serait ni étain ni plomb, mais qui ressemble à de l'argent. Prevert a donné aux Souricois des coins et des ciseaux pour qu'ils apportent des morceaux de mines l'année suivante; ailleurs, chez les Almouchiquois (sur la côte du Maine) il y a d'autres mines, mais les Souricois n'osent y aller à moins d'être accompagnés de Français pour combattre leurs ennemis. Champlain s'évertue à situer hypothétiquement ces lieux dont parle Prevert. Et il ajoute: «Tout ce païs est tres-beau, \& plat,

61 Nous aurions en ce Cap d'Or l'ancien cap Conjugon de Jacques Noël, toponyme qui serait la déformation d'un nom micmac (Ganong, Crucial Maps, IX, dans MSRC, 31 (1937), II: 124).

62 Champlain, op. cit., I: 166-172, 186-188. 
où il y a de toutes les sortes d'arbres que nous avons vues allant au premier sault de la grande Riviere de Canadas ${ }^{63}$.

A entendre de Prevert la description du littoral acadien, Champlain retourne à la préoccupation majeure du siècle précédent: le passage vers l'Asie. Les interprètes lui avaient parlé d'un grand lac, au-delà du sault Saint-Louis, qui serait peutêtre la mer d'Asie, mais Champlain souhaite qu'on puisse l'atteindre plus facilement en partant du littoral atlantique: «Ce seroit un grand bien qui pourroit trouver à la coste de la Floride quelque passage qui allast donner proche du susdict grand lac, où l'eau est sallée, tant pour la navigation des vaisseaux, lesquels ne seroient subjects à tant de perils comme ils sont en Canadas, que pour l'accourcissement du chemin de plus de trois cens lieues. ${ }^{64}$

En Acadie, beau pays et beaux arbres: ce qu'on trouvait aussi dans le Saint-Laurent, mais en Acadie il y a quantité de mines et peut-être un passage plus rapide vers la mer d'Asie; l'Acadie pourrait dispenser du Saint-Laurent, où la navigation est longue et difficile et où tant de conflits sont en jeu. En 1603, c'est l'Acadie, plus que le Saint-Laurent, qui fascine Champlain.

\section{LE BILAN DU VOYAGE DE 1603}

Le 19 juillet, Pont-Gravé et Champlain quittent l'île Percée pour retourner à Tadoussac, mais le mauvais temps leur donne l'occasion de visiter la rive nord du golfe, des Sept-Isles en amont, sans qu'on n'y ajoute toutefois à la toponymie. Le 3 août, grande fête à Tadoussac, sous la direction du chef' Begourat, pour célébrer une victoire qu'on avait remportée dans le pays des Iroquois: les Montagnaises dansent encore toutes nues devant les Français et simulent une bataille dans l'eau à coups d'avirons les unes contre les autres. C'est sur ce tableau charmant que Pont-Gravé et Champlain quittent Tadoussac le 16 août pour rejoindre Prevert à l'île Percée le surlendemain. ${ }^{65}$

63 Ibid., I: 172, 181-185.

64 Ibid., I: 170 s.

65 Ibid., I: 173-180. 
Champlain n'allait revenir dans ces parages que dans cinq ans. On emmène des sauvages en France: le fils du sagamo montagnais Begourat, que l'on confie à Pont-Gravé; une Iroquoise, capturée par les Montagnais qui voulaient la manger; Prevert en a quatre autres: un sauvage de l'Acadie, une femme et deux enfants de «Canadiens ${ }^{66}$.

Dans ce voyage de 1603 , la toponymie laurentienne ne semble faire que bien peu de progrès. Remarquons bien cependant qu'on n'a pas retrouvé la carte que Champlain aurait faite dès 1603 de ce qu'il a «veu \& recognu ${ }^{67}$. A ne lire que le seul récit de 1603 , on reste asez déçu. Des 24 noms de lieux que cite Champlain, depuis la baie de Chaleur jusqu'au sault SaintLouis ${ }^{68}$, pas un seul n'est de sa création: sept sont de l'époque de Cartier ${ }^{69}$; six sont inscrits sur la carte Levasseur de $1601^{70}$; les autres sont en usage avant Champlain: il n'en est pas l'auteur, mais il a contribué à les rendre populaires. Retenons toutefois que deux toponymes toujours vivants, sault Montmorency et lac Saint-Pierre, datent de ce voyage, mais ils n'apparaissent pas dans le récit de 1603. Quant au fleuve, il ne porte pas encore le nom de Saint-Laurent.

66 Ibid., I: 180, 188. Conduit en France par Pont-Gravé en 1603, le fils de Begourat fut installé au château Saint-Germain, en la compagnie du Dauphin (le futur Louis XIII) ; il fut surnommé petit Canada; baptisé le 9 mai 1604, il eut pour parrain Alexandre de Vendôme et, pour marraine, Catherine-Henriette de Vendôme, l'un et l'autre nés de Henri IV et de Gabrielle d'Estrées; le petit Canada mourut le 18 juin 1604 (Leymarie, Le Canada pendant la jeunesse de Louis XIII, dans Nova Francia, I, 1924: 168s.).

67 En 1632, Champlain écrit du voyage de 1603: «Je fis dès lors un petit discours avec la carte exacte de tout ce que j'avois veu \& recognu » (Champlain, op. cit., III: 317).

${ }^{68} \mathrm{Ce}$ sont, en remontant le fleuve: Baie de Chaleur, île Bonaventure, île Percée, baie des Molues, Gaspé, cap L'Evesque, Matane, le Pic (pour le Bic: ce n'est pas ici une faute d'impression comme le prétend Biggar, ibid., I: 95 , n. 5 ; car la carte Levasseur donnait aussi Pic); rivière SteMarguerite, Lesquemin, Tadoussac, Pointe-St-Mathieu, Pointe-de-tous-lesDiables, Saguenay, île aux Lièvres, île aux Coudres, île d'Orléans, rivière de Canada, Québec, rivière Ste-Croix, rivière Batiscan, île St-Eloi, TroisRivières, rivière des Iroquois.

69 Baie de Chaleur, Saguenay, île aux Lièvres, île aux Coudres, île d'Orléans, rivière de Canada et rivière Ste-Croix.

70 Gaspé, Pic (pour Bic), Tadoussac, Saguenay, Québec, Trois-Rivières. 
Aucun progrès dans la toponymie acadienne. On s'y attendait, puisque Pont-Gravé ne fait aucune expédition de ce côté en 1603 et que le voyage de Prevert n'est que celui d'un prospecteur. Le récit de 1603 nous transmet quelques toponymes, déjà en usage: Tregate, Misamichy, rivière Souricoua, île SaintLaurent, Cap-Breton, Arcadie ou Acadie, île Saint-Jean ${ }^{71}$.

De la «tabagie» de 1603 il faut enregistrer un résultat de haute importance: les indigènes du Saint-Laurent consentent à ce que les Français peuplent le pays. La déclaration du chef Anadabijou (si elle est exactement rapportée par Champlain) est formelle: "Qu'il estoit fort aise que sadicte Majesté peuplast leur terre. »72 Quand les Hollandais s'établiront à New-York, ils achèteront des indigènes l'île de Manhatte; quand William Penn voudra coloniser sa forêt, la Pennsylvanie, il en achètera aussi le sol; ici, à Tadoussac en 1603, point d'achat, point de cession ni de traité rédigé en bonne et due forme: les Français reçoivent, au cours d'une assemblée solennelle, l'autorisation générale d'habiter le pays. Il n'y a pas eu spoliation de l'indigène.

Lors de cette «tabagie », on constate qu'une ligue couvre la vallée depuis le golfe jusqu'aux Grands Lacs; elle assure aux Français un immense territoire de traite, mais à condition évidemment qu'ils prennent position contre les Iroquois. Condition inévitable: dès le début du dix-septième siècle, les Français sont engagés dans un conflit dont Champlain n'est aucunement responsable. La «tabagie » de 1603 établit que les indigènes comptent depuis quelque temps sur les Français; ceux-ci s'engagent, sans que Champlain n'y soit pour rien, à faire entendre raison aux Iroquois ou à les réduire par les armes. Puisque l'état de guerre existait déjà en 1542 entre Français et Iroquois, 1603 ne fait que marquer une reprise du conflit: la guerre ne débute pas, elle continue.

Notons enfin que les Français ont refait un inventaire du Saint-Laurent. Le pays offre de nombreux avantages: dans la région de Québec, terres unies et plaisantes qui seraient aussi

${ }^{71}$ Ile Saint-Laurent deviendra Cap-Breton; île Saint-Jean sera remplacé par île du Prince-Edouard.

72 Champlain, op. cit., I: 101. 
bonnes que celles de France si on les cultivait; en amont, belles terres encore, noires, sans rochers, riches en bois; climat plus tempéré dans la région des Trois-Rivières avec des terres encore meilleures autour du lac Saint-Pierre; abondance de bonnes prairies dans la région de Montréal. Champlain en 1603 n'a pas été particulièrement frappé par le site de Québec; le seul endroit où il voudrait voir surgir une habitation, c'est aux Trois-Rivières et encore ne serait-elle là que pour servir d'étape de sécurité dans la route des fourrures. Dans cette vallée du Saint-Laurent, l'espérance est plutôt incertaine de trouver des mines; certes, toujours à l'affût de découvertes minières, Champlain a cru voir au rocher de Québec des diamants meilleurs que ceux d'Alençon (ces mêmes diamants qui avaient trompé Cartier) ; en passant par les Algonquins et les «bons Irocois» au nord-ouest du sault Saint-Louis, on aurait accès à une mine de franc cuivre, mais cela demeurait fort aléatoire. A cette incertitude s'ajoute un état permanent de guerre qui complique les échanges commerciaux.

A l'égard du Saint-Laurent en 1603, il reste en définitive une certaine déception, du pessimisme: serait-ce que Champlain a lu le récit des voyages de Cartier ? Ou que, tout simplement, le Canada a toujours mauvaise presse depuis l'échec de Roberval ? Par contre, l'Acadie exerce une forte attirance chez Champlain: il y a là, à ce qu'on lui a dit, un beau pays, de beaux arbres (ce qu'on trouvait aussi dans le Saint-Laurent), mais, de plus, quantité de mines et peut-être un passage plus rapide vers la mer d'Asie. La relation de Champlain fait pressentir que l'Acadie dispensera peut-être du Saint-Laurent et que, si les Français reviennent, ce sera pour courir après les promesses de la mystérieuse Acadie.

\section{L'ANGLETERRE N'EST PAS PLUS HEUREUSE}

La France depuis Cartier n'a subi que des déboires, alors que l'Espagne et le Portugal ont fermement établi leur civilisation, du détroit de Magellan à la Floride. On serait tenté de conclure que la France est inapte à la colonisation américaine. 
Or, perdu pour la France, le seizième siècle l'est aussi pour l'Angleterre. Comme la France, l'Angleterre a connu ses périodes d'indifférence à l'égard de l'Amérique: Cabot a déçu parce qu'il revenait sans métaux précieux. Henri VII et Henri VIII ont d'abord à assurer l'unité de leur pays; ils doivent aussi éviter de heurter l'Espagne dans son expansion impérialiste; enfin, la vie économique de l'Angleterre est encore centrée sur l'Europe, en particulier sur la Méditerranée. L'Angleterre soutient quelques voyages hauturiers, mais elle cherche alors à étendre son commerce plutôt qu'à fonder une NouvelleAngleterre.

Tout change sous le long règne d'Elizabeth (1558-1603): le centre d'intérêt économique n'est plus la Méditerranée, mais l'Atlantique; rapidement industrialisée, l'Angleterre veut de nouveaux marchés et des sources nouvelles et moins coûteuses d'approvisionnement; la montée des classes moyennes, que dominent les marchands et manufacturiers, renforcit l'État; le protestantisme fait désormais de l'Angleterre l'adversaire des nations catholiques, en particulier de celle qui est la plus énergique à soutenir la Papauté, l'Espagne. La reine Elizabeth tentera donc de faire échec au Roi Très Catholique sur les lieux même d'où il tire sa toute-puissance, en Amérique; elle tentera de s'enrichir des mêmes richesses et, en même temps, de trouver une voie rapide vers le plantureux commerce d'Orient.

Quand l'Angleterre d'Elizabeth se tourne définitivement vers l'Amérique, elle dispose d'une marine qui, établie sous Henri VII, est devenue imposante; de marins, ces célèbres seadogs, qui vont se lancer dans des aventures extraordinaires: ce Frobisher qui de 1576 à 1578 cherche la route d'Asie à travers les glaces du nord-ouest, ces corsaires de grande classe (John Hawkins et Francis Drake) qui vont à plusieurs reprises frapper au cœur du monde hispano-américain pour revenir chargés de dépouilles. De zélés propagandistes vont tenir leurs compatriotes en alerte: Richard Hakluyt l'aîné et son cousin le révérend Richard Hakluyt (dit le jeune) vont mettre la colonisation américaine à la mode par leurs tableaux enthousiastes, par des 
publications qui sont des programmes à application immédiate. La conjoncture est favorable aux grands entrepreneurs de colonisation.

Sans pour autant qu'ils connaissent plus de succès que la France. Humphrey Gilbert part en novembre 1578 avec sept navires, mais, comme La Roche la même année, il doit retourner à son point de départ sans avoir touché son objectif. A la suite d'une reconnaissance faite par Simon Ferdinando en 1579 sur la côte de Norembègue, Gilbert fait visiter la mêrne côte par John Walker : celui-ci se rend sur la rivière Penobscot, découvre une mine d'argent et rapporte de riches fourrures. En 1583, Gilbert arrive à Terre-Neuve avec une flotte de cinq navires, il prend officiellement possession de l'île, puis se met en route pour la mystérieuse Norembègue: une tempête le détourne de l'objectif, une autre tempête le fait sombrer. ${ }^{73}$

Son demi-frère, Walter Ralegh, prend la relève: il obtient en 1584 un domaine de 200 lieues là où il s'établira ${ }^{74}$; ses fondés de pouvoir, Philip Amadas et Arthur Barlowe, choisissent une région qu'on appellera Virginie; en 1585, Richard Grenville y conduit une flotte de sept navires avec 107 personnes: il établit son monde sur l'île Roanoke, le laisse sous les ordres de Ralph Lane et rentre en Angleterre. Bonnes au début, les relations avec les indigènes se détériorent peu à peu ${ }^{75}$, la colonie subit la guerre, l'approvisionnement devient difficile; quand Drake passe par là en 1586, les colons se font rapatrier et l'indigène Manteo, resté fidèle, rentre en Angleterre avec eux. Ils venaient de partir, lorsqu'un navire de ravitaillement se présente, qui ne trouvant plus personne reprend la route de l'Angleterre. Quinze jours plus tard arrive Grenville avec trois navires: pour 106s., 171.

73 Winsor, Narrative and Critical History of North America, III:

${ }_{74}$ Texte de la charte de 1584, dans Documents of American History (éd. Commager), $6 \mathrm{~s}$.

75 En 1584, on avait emmené en Angleterre deux indigènes pour leur apprendre l'anglais; en 1585, ils font partie de la colonie, mais l'un d'eux fit tout pour détourner des Anglais ses compatriotes: Domagaya et Taignoagny, que Cartier avait emmenés en France pour les préparer au rôle d'interprètes, s'étaient comportés de la même façon à Stadaconé. 
s'assurer la possession des lieux, il y dépose quinze hommes avec des provisions pour deux ans et rentre chez lui. ${ }^{76}$

Ralegh se remet à l'œuvre; cette fois c'est dans la baie de Chesapeake, où les Espagnols en 1570 avaient tenté un établissement 77, qu'il veut fonder une Nouvelle-Angleterre ou, comme il l'appelle, «la ville de Ralegh en Virginie ». Choisi pour gouverneur, John White s'embarque en 1587 avec 150 personnes dont 17 femmes: on arrête à l'île Roanoke pour chercher en vain les 15 hommes de 1586, puis, à cause de la saison, on décide de s'y établir; c'est là qu'on baptise l'indigène Manteo (ce serait le premier baptême d'indigène au nord de la Floride) et on le nomme seigneur du pays; c'est là que la fille du gouverneur White met au monde une enfant, le 18 août 1587, qu'on appelle Virginie et qui serait depuis les Vikings le premier enfant européen à naître au nord de la Floride. Rentré en Angleterre, White ne réussit à revenir qu'en 1590: il n'y avait plus personne ${ }^{78}$.

L'échec de 1590 marque un arrêt d'une douzaine d'années dans les tentatives de colonisation anglaise: la guerre contre l'Espagne, les profits rapides de la course en mer, l'intérêt de Ralegh pour l'Amérique du Sud, la reprise du commerce avec l'Europe et la fondation de la Compagnie des Indes orientales relèguent à l'arrière-plan une Nouvelle-Angleterre américaine.

En 1602, quatre ans après le départ de La Roche pour l'île de Sable et deux ans après la fondation de Chauvin à Tadoussac, des Anglais se remettent à l'œuvre. Sans l'autorisation de Ralegh, Bartholomew Gosnold part sur le Concord avec 32 personnes. Muni de la relation de Verrazano, comme le Florentin il suit la route directe au travers de l'Atlantique (ce qu'on n'avait pas fait depuis le voyage de Ribault en 1562) ; le 14 mai, il arrive en vue d'une côte qu'il appelle Northland

76 Winsor, op. cit., III : 110-113, 114.

77 W. Lowery, The Spanish Settlements, 1562-1574, 359ss.

78 Winsor, op. cit., III: 113-116, 129. Divers indices permettent de soutenir que des colons de Roanoke se seraient mêlés aux autochtones; les indigènes Hatteras, aux yeux gris, se réclamaient d'ancêtres blancs; en 1607 , on y rencontrera un jeune indigène à peau blanche et à cheveux blonds. 
(c'est Casco Bay, dans le Maine); il s'arrête à un cap qu'il nomme Savage Rock et qui est le cap Neddock; il y rencontre des indigènes à bord d'une embarcation basque; ceux-ci, dont certains portent des articles vestimentaires d'Europe, lui font un dessin du littoral. Il contourne un cap auquel il donne le nom de Cape Cod, il s'arrête au large de la baie de Newport, sur une île appelée Elizabeth (qui est aujourd'hui Cuttyhunk) et y bâtit un petit fort et une maison. Gosnold se trouvait dans le pays du Refuge où Verrazano avait séjourné en 1524. Sur la terre ferme, appelée Mayne, on fit des expéditions pour amasser du cèdre et du sassafras, mais quand vint le moment pour Gosnold de se séparer de ses colons, la mésentente obligea tout le monde à rentrer en Angleterre au cours de cette même année $1602 .^{79}$

Ralegh ne perd pas de vue pour autant ses lettres qui lui permettent d'établir la Virginie et il envoie deux expéditions en 1603: sur l'Elizabeth, Bartholomew Gilbert se dirige vers la Virginie; il y est tué par les indigènes et l'on revient sans avoir rien fait; avec le Speedwell et le Discoverer, Martin Pring se porte plus au nord: le 2 juin 1603, alors que Pont-Gravé et Champlain sont dans le Saint-Laurent, Pring arrive en vue des côtes du Maine, à l'est de la baie de Penobscot dont une île reçoit le nom de $F o x$; descendant vers le sud, il retrouve Savage Rock, il pénètre dans la baie de Massachusetts, puis séjourne six semaines dans la baie de Plymouth qu'il appelle Whitson: il y précédait de deux ans Champlain et de dix-sept ans les célèbres Pilgrims. Pring élève une barricade, fait des semailles pour éprouver la fertilité du sol, amasse du sassafras; les indigènes feignent d'abord de l'amitié, puis se déclarent ouvertement contre la colonie: tout le monde est de retour en Angleterre en octobre 1603.80 C'était exactement l'année où la France abandonnait pour un temps la vallée du Saint-Laurent et, pour de bon, l'île de Sable.

${ }^{79}$ Récit de John Brereton publié en 1602 et reproduit dans ForeRunners of the Pilgrims, I: $30-41$; relation de Gabriel Archer, ibid., 43-54; lettre de Gosnold à son père, 1602 , ibid., 55s.; voir aussi Winsor, op. cit., III : $172 \mathrm{~s}$.

80 Journal du voyage de Pring, dans Fore-Runners, I: 60-68; voir aussi Winsor, op. cit., III: $173 \mathrm{~s}$. 
Ces multiples tentatives de l'Angleterre ne donnaient qu'un seul résultat immédiat et de quelque durée: cette toponymie anglaise qui, au sud de la Nouvelle-Écosse, parvient enfin à supplanter une toponymie espagnole vieille de soixante-quinze ans ${ }^{81}$. Bien que l'Angleterre soit alors pourvue d'une marine formidable que dirigent de vieux loups de mer, bien qu'elle soit riche d'un commerce étendu et jette son dévolu sur une région où le climat est bien plus accueillant que celui du Saint-Laurent, elle échoue toujours, comme la France, dans ses tentatives de fonder une colonie américaine. Ou bien l'Europe ne s'applique pas sérieusement à la colonisation, ou bien elle n'a pas encore appris à vivre en Amérique du Nord.

\section{MARCEL TRUDEL, directeur \\ Institut d'Histoire \\ Université Laval (Québec)}

81 Cette première toponymie anglaise, au sud de la Nouvelle-Ecosse, est la suivante:

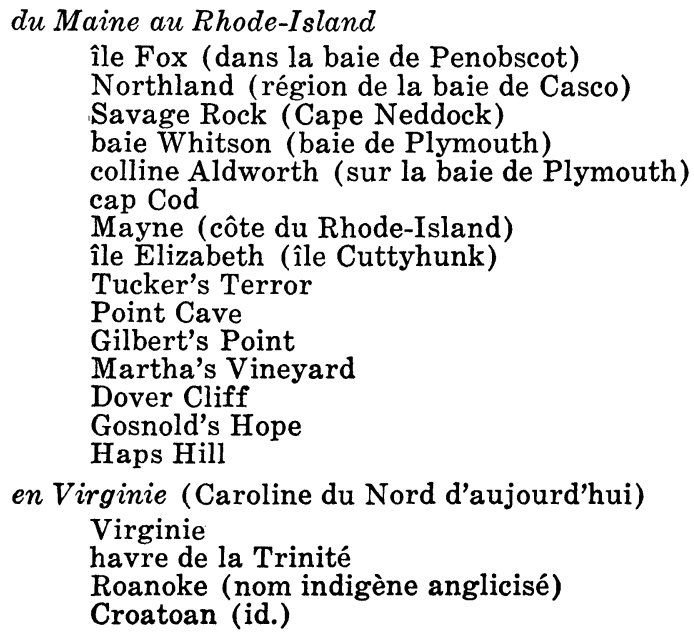

